\title{
Serum Tumor Marker Test
}

National Cancer Institute

\section{Source}

National Cancer Institute. Serum Tumor Marker Test. NCI Thesaurus. Code 693025.

A laboratory test in which a monoclonal antibody is used to detect a tumor-specific

marker in a blood sample. 